\title{
Travelling Wave Solutions for the Coupled IBq Equations by Using the tanh-coth Method
}

\author{
Omer Faruk Gozukizil $^{1}$ and Samil Akcagil $^{2}$ \\ ${ }^{1}$ Department of Mathematics, Sakarya University, 9054187 Sakarya, Turkey \\ ${ }^{2}$ Vocational School of Pazaryeri, Bilecik Şeyh Edebali University, 9011800 Bilecik, Turkey \\ Correspondence should be addressed to Omer Faruk Gozukizil; farukg@sakarya.edu.tr
}

Received 4 May 2013; Accepted 9 December 2013; Published 19 January 2014

Academic Editor: Ch. Tsitouras

Copyright (c) 2014 O. F. Gozukizil and S. Akcagil. This is an open access article distributed under the Creative Commons Attribution License, which permits unrestricted use, distribution, and reproduction in any medium, provided the original work is properly cited.

\begin{abstract}
Based on the availability of symbolic computation, the tanh-coth method is used to obtain a number of travelling wave solutions for several coupled improved Boussinesq equations. The abundant new solutions can be seen as improvement of the previously known data. The obtained results in this work also demonstrate the efficiency of the method.
\end{abstract}

\section{Introduction}

We consider the following two coupled improved Boussinesq (IMBq) equations of Sobolev type:

$$
\begin{gathered}
u_{t t}-\alpha^{2} u_{x x t t}-u_{x x}=f(u, w)_{x x}, \quad x \in \mathbb{R}, t>0, \\
w_{t t}-\alpha^{2} w_{x x t t}-w_{x x}=g(u, w)_{x x}, \quad x \in \mathbb{R}, t>0,
\end{gathered}
$$

where $f$ and $g$ are given nonlinear functions, $u(x, t)$ and $w(x, t)$ are unknown functions, and $\alpha$ is a constant that has been derived to describe bidirectional wave propagation in several studies, for instance, in a Toda lattice model with a transversal degree of freedom, in a two-layered lattice model, and in a diatomic lattice. Dé Godefroy has studied (1) as the Cauchy problem under certain conditions and showed that the solution for the Cauchy problem of this system blows up in finite time [1]. Wang and Li have considered the Cauchy problem for (1), proved the existence and uniqueness of the global solution, and given sufficient conditions of blow-up of the solution in finite time by convex methods [2]. The Cauchy problem for (1) has been studied and established the conditions for the global existence and finite-time blow-up of solutions in Sobolev spaces $H^{s} \times H^{s}$ for $s>1 / 2$ [3]. For more information, we refer the reader to [3] and references therein.
Chen and Zhang have considered the initial boundary value problem for the system of the generalized IMBq type equations:

$$
\begin{gathered}
u_{t t}-A u_{x x}-B u_{x x t t}=m(w)_{x x}, \quad 0<x<l_{1}, t>0, \\
w_{t t}-B w_{x x t t}=n(w)_{x x}, \quad 0<x<l_{1}, t>0,
\end{gathered}
$$

where $u(x, t)$ and $w(x, t)$ are unknown functions, $A, B>0$ are constants, and $m$ and $n$ are the given nonlinear functions. As a result, they have proved the existence and uniqueness of the global generalized solution and the global classical solution [4].

Rosenau is concerned with the problem of how to describe the dynamics of a dense lattice via the system

$$
\begin{gathered}
u_{t t}=(u+2 \alpha u w)_{x x}+\frac{1}{12} u_{x x t t} \\
w_{t t}=\left(2 w+\beta w^{2}+\alpha u^{2}\right)_{x x}+\frac{1}{12} w_{x x t t}
\end{gathered}
$$

where $\alpha, \beta>0$ are constants, and pointed out that (3) was a convenient vehicle to study the dynamics of a dense lattice [5].

In [6], a transversal degree of freedom was introduced in the Toda lattice. For different order of magnitude of the longitudinal and transversal strains, coupled and uncoupled 
equations for these fields were derived in the discrete case as well as the continuum limit. The system

$$
\begin{gathered}
\frac{\rho}{\alpha} u_{t t}=\beta u_{x x}-\frac{\beta^{2}}{2}\left(u^{2}\right)_{x x}+\frac{\beta}{2}\left(w^{2}\right)_{x x}+\frac{\rho}{\alpha} \frac{l^{2}}{12} u_{x x t t}, \\
\frac{\rho}{\alpha} w_{t t}=\beta(u w)_{x x}+\frac{\rho}{\alpha} \frac{l^{2}}{12} w_{x x t t}
\end{gathered}
$$

has been obtained for the longitudinal and transversal strains. $l$ and $1 / b$ are characteristic lengths in the model, $\beta$ is their ratio (i.e., $\beta=l b$ ), $\rho$ denotes the linear mass density, and $\alpha>0$ is a constant. Besides, travelling wave solutions and numerical solutions of the system have been found.

Turitsyn has proved the existence of blow-up for the continuum limit model of the Toda lattice with a transversal degree of freedom analytically and analysed the coupled IBq equations

$$
\begin{gathered}
u_{t t}=\beta u_{x x}-\frac{\beta}{2}\left(u^{2}\right)_{x x}+\frac{1}{2}\left(w^{2}\right)_{x x}+\alpha^{2} u_{x x t t}, \\
w_{t t}=(u w)_{x x}+\alpha^{2} w_{x x t t} .
\end{gathered}
$$

Turitsyn has showed that the sufficient criterion for the collapse in this model may be formulated as the requirement of nonpositively of the system Hamiltonian [7].

Pego et al. have studied the stability of solitary waves of two coupled improved Boussinesq equations:

$$
\begin{gathered}
u_{t t}=u_{x x}-\beta\left(u^{2}\right)_{x x}+\left(w^{2}\right)_{x x}+u_{x x t t}, \\
w_{t t}=w_{x x}+2(u w)_{x x}+w_{x x t t},
\end{gathered}
$$

which model weakly nonlinear vibrations in a cubic lattice. They have encountered this system for which the classic method fails [8].

Fan and Tian have expanded the model in [6] and considered the following general Toda dense lattice equation which is governed by the following coupled improved Boussinesq equation:

$$
\begin{gathered}
u_{t t}=\beta u_{x x}-\frac{\beta}{2}\left(u^{2}\right)_{x x}+\frac{\beta}{2}\left(w^{2}\right)_{x x}+\alpha^{2} u_{x x t t}, \\
w_{t t}=\gamma(u w)_{x x}+\rho\left(w^{2}\right)_{x x}+\alpha^{2} w_{x x t t} .
\end{gathered}
$$

They have studied compacton solutions and multicompacton solutions of transversal degree of freedom by direct sine and cosine method and found peakon solutions. Moreover, they have presented more solitary wave solutions for a special case of (7) using improved sin-cos and improved sinh-cosh method [9].

The paper is arranged as follows. We described the outline of the tanh-coth method in the following section and derived various exact travelling wave solutions of the coupled IBq equations (3), (4), (5), (6) and (7) in the next sections. Finally, we summarized our conclusions in the last section.

\section{Outline of the tanh-coth Method}

Wazwaz has summarized the tanh-coth method [10] in the following manner. (i) First, consider a general form of nonlinear equation

$$
P\left(u, u_{t}, u_{x}, u_{x x}, \ldots\right)=0 .
$$

(ii) To find the traveling wave solution of (8), the wave variable $\xi=x-V t$ is introduced so that

$$
u(x, t)=U(\mu \xi) .
$$

Based on this, one may use the following changes:

$$
\begin{gathered}
\frac{\partial}{\partial t}=-V \frac{d}{d \xi}, \\
\frac{\partial}{\partial x}=\mu \frac{d}{d \xi}, \\
\frac{\partial^{2}}{\partial x^{2}}=\mu^{2} \frac{d^{2}}{d \xi^{2}}, \\
\frac{\partial^{3}}{\partial x^{3}}=\mu^{3} \frac{d^{3}}{d \xi^{3}},
\end{gathered}
$$

and so on for other derivatives. Using (10) changes the PDE (8) to an ODE:

$$
Q\left(U, U^{\prime}, U^{\prime \prime}, \ldots\right)=0
$$

(iii) If all terms of the resulting ODE contain derivatives in $\xi$, then by integrating this equation, and by considering the constant of integration to be zero, one obtains a simplified ODE.

(iv) A new independent variable

$$
Y=\tanh (\mu \xi)
$$

is introduced which leads to the change of derivatives:

$$
\begin{gathered}
\frac{d}{d \xi}=\mu\left(1-Y^{2}\right) \frac{d}{d Y} \\
\frac{d^{2}}{d \xi^{2}}=-2 \mu^{2} Y\left(1-Y^{2}\right) \frac{d}{d Y}+\mu^{2}\left(1-Y^{2}\right)^{2} \frac{d^{2}}{d Y^{2}} \\
\frac{d^{3}}{d \xi^{3}}=2 \mu^{3}\left(1-Y^{2}\right)\left(3 Y^{2}-1\right) \frac{d}{d Y} \\
-6 \mu^{3} Y\left(1-Y^{2}\right)^{2} \frac{d^{2}}{d Y^{2}}+\mu^{3}\left(1-Y^{2}\right)^{3} \frac{d^{3}}{d Y^{3}} \\
\frac{d^{4}}{d \xi^{4}}=-8 \mu^{4} Y\left(1-Y^{2}\right)\left(3 Y^{2}-2\right) \frac{d}{d Y} \\
+4 \mu^{4}\left(1-Y^{2}\right)^{2}\left(9 Y^{2}-2\right) \frac{d^{2}}{d Y^{2}} \\
-12 \mu^{4} Y\left(1-Y^{2}\right)^{3} \frac{d^{3}}{d Y^{3}} \\
+\mu^{4}\left(1-Y^{2}\right)^{4} \frac{d^{4}}{d Y^{4}},
\end{gathered}
$$

where other derivatives can be derived in a similar manner. 
(v) The ansatz of the form

$$
U(\mu \xi)=S(Y)=\sum_{k=0}^{M} a_{k} Y^{k}+\sum_{k=1}^{M} b_{k} Y^{-k}
$$

is introduced where $M$ is a positive integer, in most cases, that will be determined. If $M$ is not an integer, then a transformation formula is used to overcome this difficulty. Substituting (13) and (14) into the ODE (11) yields an equation in powers of $Y$.

(vi) To determine the parameter $M$, the linear terms of the highest order in the resulting equation with the highest order nonlinear terms are balanced. With $M$ determined, one collects all coefficients of powers of $Y$ in the resulting equation where these coefficients have to vanish. This will give a system of algebraic equations involving the $a_{k}$ and $b_{k},(k=0, \ldots, M), V$, and $\mu$. Having determined these parameters, knowing that $M$ is a positive integer in most cases, and using (14), one obtains an analytic solution in a closed form.

\section{The Travelling Wave Solutions}

3.1. The First Coupled. We first consider the following two coupled IBq equations:

$$
\begin{gathered}
u_{t t}=(u+2 \alpha u w)_{x x}+\frac{1}{12} u_{x x t t} \\
w_{t t}=\left(2 w+\beta w^{2}+\alpha u^{2}\right)_{x x}+\frac{1}{12} w_{x x t t} .
\end{gathered}
$$

Using the wave variable $\xi=x-V t$, then by integrating this equation twice and considering the constants of integration to be zero, the system (15) is carried to a system of ODEs:

$$
\begin{gathered}
12\left(V^{2}-1\right) U-24 \alpha U W-V^{2} U^{\prime \prime}=0, \\
12\left(V^{2}-2\right) W-12 \beta W^{2}-12 \alpha U^{2}-V^{2} W^{\prime \prime}=0 .
\end{gathered}
$$

Balancing $V^{2} U^{\prime \prime}$ with $U W$ and $V^{2} W^{\prime \prime}$ with $U^{2}$ in (16) gives

$$
\begin{gathered}
M+2=M+N, \\
2 M=N+2,
\end{gathered}
$$

so that $M=N=2$. We consider solutions in the form

$$
\begin{aligned}
& U(\mu \xi)=S(Y)=\sum_{k=0}^{2} a_{k} Y^{k}+\sum_{k=1}^{2} b_{k} Y^{-k} \\
& W(\mu \xi)=S(Y)=\sum_{k=0}^{2} c_{k} Y^{k}+\sum_{k=1}^{2} d_{k} Y^{k}
\end{aligned}
$$

Substituting (18) into the two components of (16) and collecting the coefficients of $Y$ give two systems of algebraic equations for $a_{0}, a_{1}, a_{2}, b_{1}, b_{2}, c_{0}, c_{1}, c_{2}, d_{1}, d_{2}, V$, and $\mu$. Solving these systems together leads to the following sets:

$$
\begin{aligned}
& a_{0}=-\frac{a_{2}}{3}, \quad a_{2}=\frac{3 \sqrt{\alpha \beta\left(V^{4}-3 V^{2}+2\right)}}{4 \alpha^{2}}, \\
& c_{0}=\frac{\beta+4 \alpha}{8 \alpha(\alpha-\beta)}, \quad c_{2}=\frac{-3 \beta}{8 \alpha(\alpha-\beta)}, \\
& V=\frac{6}{\sqrt{-6 \mu^{2}+18}}, \quad \mu=\frac{\sqrt{3 \beta(-3 \beta+4 \alpha)}}{-3 \beta+4 \alpha}, \\
& a_{1}=b_{1}=b_{2}=c_{1}=d_{1}=d_{2}=0, \\
& a_{0}=-2 b_{2}, \quad a_{2}=\frac{9 \beta^{2}(2 \alpha-\beta)}{1024 \alpha^{3} b_{2}(\alpha-\beta)^{2}}, \\
& b_{2}=\frac{3 \sqrt{\alpha \beta\left(V^{4}-3 V^{2}+2\right)}}{16 \alpha^{2}}, \quad c_{0}=\frac{-3 \beta+8 \alpha}{16 \alpha(\alpha-\beta)}, \\
& c_{2}=\frac{3 \beta}{32 \alpha(\alpha-\beta)}, \quad d_{2}=c_{2}, \\
& V=\frac{6}{\sqrt{24 \mu^{2}+18}}, \quad \mu=\frac{\sqrt{\beta(9 \beta-12 \alpha)}}{-6 \beta+8 \alpha}, \\
& a_{1}=b_{1}=c_{1}=d_{1}=0, \\
& a_{0}=\frac{2 b_{2}}{3}, \quad a_{2}=\frac{9 \beta^{2}(2 \alpha-\beta)}{1024 \alpha^{3} b_{2}(\alpha-\beta)^{2}}, \\
& b_{2}=\frac{3 \sqrt{\alpha \beta\left(V^{4}-3 V^{2}+2\right)}}{16 \alpha^{2}}, \quad c_{0}=\frac{-\beta+8 \alpha}{16 \alpha(\alpha-\beta)}, \\
& c_{2}=\frac{-3 \beta}{32 \alpha(\alpha-\beta)}, \quad d_{2}=c_{2}, \\
& V=\frac{6}{\sqrt{-24 \mu^{2}+18}}, \quad \mu=\frac{\sqrt{3 \beta(-3 \beta+4 \alpha)}}{-6 \beta+8 \alpha}, \\
& a_{1}=b_{1}=c_{1}=d_{1}=0, \\
& a_{0}=\frac{-9 \beta^{2}(2 \alpha-\beta)}{64 \alpha^{3} a_{2}(\alpha-\beta)^{2}}, \quad a_{2}=\frac{3 \sqrt{\alpha \beta\left(V^{4}-3 V^{2}+2\right)}}{4 \alpha^{2}} \text {, } \\
& c_{0}=\frac{-3 \beta+4 \alpha}{8 \alpha(\alpha-\beta)}, \quad c_{2}=\frac{3 \beta}{8 \alpha(\alpha-\beta)}, \\
& V=\frac{6}{\sqrt{6 \mu^{2}+18}}, \quad \mu=\frac{\sqrt{\beta(9 \beta-12 \alpha)}}{-3 \beta+4 \alpha}, \\
& a_{1}=b_{1}=b_{2}=c_{1}=d_{1}=d_{2}=0 \text {. }
\end{aligned}
$$


Consequently, we obtain the following travelling wave solutions:

$$
\begin{aligned}
u_{1}(x, t)= & -\frac{\sqrt{\alpha \beta\left(V^{4}-3 V^{2}+2\right)}}{4 \alpha^{2}} \\
& +\frac{3 \sqrt{\alpha \beta\left(V^{4}-3 V^{2}+2\right)}}{4 \alpha^{2}} \tanh ^{2} \frac{\sqrt{3 \beta(-3 \beta+4 \alpha)}}{-3 \beta+4 \alpha} \\
& \times\left(x-\frac{6}{\sqrt{-6 \mu^{2}+18}} t\right)
\end{aligned}
$$$$
w_{1}(x, t)=\frac{\beta+4 \alpha}{8 \alpha(\alpha-\beta)}
$$$$
-\frac{3 \beta}{8 \alpha(\alpha-\beta)} \tanh ^{2} \frac{\sqrt{3 \beta(-3 \beta+4 \alpha)}}{-3 \beta+4 \alpha}
$$$$
\times\left(x-\frac{6}{\sqrt{-6 \mu^{2}+18}} t\right)
$$$$
u_{2}(x, t)=-\frac{3 \sqrt{\alpha \beta\left(V^{4}-3 V^{2}+2\right)}}{8 \alpha^{2}}
$$$$
+\frac{9 \beta^{2}(2 \alpha-\beta)}{1024 \alpha^{3} b_{2}(\alpha-\beta)^{2}} \tanh ^{2} \frac{\sqrt{\beta(9 \beta-12 \alpha)}}{-6 \beta+8 \alpha}
$$$$
\times\left(x-\frac{6}{\sqrt{24 \mu^{2}+18}} t\right)
$$$$
+\frac{3 \sqrt{\alpha \beta\left(V^{4}-3 V^{2}+2\right)}}{16 \alpha^{2}} \operatorname{coth}^{2} \frac{\sqrt{\beta(9 \beta-12 \alpha)}}{-6 \beta+8 \alpha}
$$$$
\times\left(x-\frac{6}{\sqrt{24 \mu^{2}+18}} t\right)
$$$$
w_{2}(x, t)=\frac{-3 \beta+8 \alpha}{16 \alpha(\alpha-\beta)}
$$$$
+\frac{3 \beta}{32 \alpha(\alpha-\beta)} \tanh ^{2} \frac{\sqrt{\beta(9 \beta-12 \alpha)}}{-6 \beta+8 \alpha}
$$$$
\times\left(x-\frac{6}{\sqrt{24 \mu^{2}+18}} t\right)
$$

$$
\begin{aligned}
& +\frac{3 \beta}{32 \alpha(\alpha-\beta)} \operatorname{coth}^{2} \frac{\sqrt{\beta(9 \beta-12 \alpha)}}{-6 \beta+8 \alpha} \\
& \times\left(x-\frac{6}{\sqrt{24 \mu^{2}+18}} t\right) \\
& u_{3}(x, t)=\frac{\sqrt{\alpha \beta\left(V^{4}-3 V^{2}+2\right)}}{8 \alpha^{2}} \\
& +\frac{9 \beta^{2}(2 \alpha-\beta)}{1024 \alpha^{3} b_{2}(\alpha-\beta)^{2}} \tanh ^{2} \frac{\sqrt{3 \beta(-3 \beta+4 \alpha)}}{-6 \beta+8 \alpha} \\
& \times\left(x-\frac{6}{\sqrt{-24 \mu^{2}+18}} t\right) \\
& +\frac{3 \sqrt{\alpha \beta\left(V^{4}-3 V^{2}+2\right)}}{16 \alpha^{2}} \operatorname{coth}^{2} \frac{\sqrt{3 \beta(-3 \beta+4 \alpha)}}{-6 \beta+8 \alpha} \\
& \times\left(x-\frac{6}{\sqrt{-24 \mu^{2}+18}} t\right)
\end{aligned}
$$$$
w_{3}(x, t)=\frac{-\beta+8 \alpha}{16 \alpha(\alpha-\beta)}
$$$$
-\frac{3 \beta}{32 \alpha(\alpha-\beta)} \tanh ^{2} \frac{\sqrt{3 \beta(-3 \beta+4 \alpha)}}{-6 \beta+8 \alpha}
$$$$
\times\left(x-\frac{6}{\sqrt{-24 \mu^{2}+18}} t\right)
$$$$
-\frac{3 \beta}{32 \alpha(\alpha-\beta)} \operatorname{coth}^{2} \frac{\sqrt{3 \beta(-3 \beta+4 \alpha)}}{-6 \beta+8 \alpha}
$$$$
\times\left(x-\frac{6}{\sqrt{-24 \mu^{2}+18}} t\right)
$$$$
u_{4}(x, t)=\frac{-9 \beta^{2}(2 \alpha-\beta)}{64 \alpha^{3} a_{2}(\alpha-\beta)^{2}}
$$$$
+\frac{3 \sqrt{\alpha \beta\left(V^{4}-3 V^{2}+2\right)}}{4 \alpha^{2}} \tanh ^{2} \frac{\sqrt{\beta(9 \beta-12 \alpha)}}{-3 \beta+4 \alpha}
$$$$
\times\left(x-\frac{6}{\sqrt{6 \mu^{2}+18}} t\right)
$$ 


$$
\begin{aligned}
w_{4}(x, t)= & \frac{-3 \beta+4 \alpha}{8 \alpha(\alpha-\beta)}+\frac{3 \beta}{8 \alpha(\alpha-\beta)} \tanh ^{2} \frac{\sqrt{\beta(9 \beta-12 \alpha)}}{-3 \beta+4 \alpha} \\
& \times\left(x-\frac{6}{\sqrt{6 \mu^{2}+18}} t\right) .
\end{aligned}
$$

3.2. The Second Coupled. We next consider the system of equations

$$
\begin{gathered}
\frac{\rho}{\alpha} u_{t t}=\beta u_{x x}-\frac{\beta^{2}}{2}\left(u^{2}\right)_{x x}+\frac{\beta}{2}\left(w^{2}\right)_{x x}+\frac{\rho}{\alpha} \frac{l^{2}}{12} u_{x x t t}, \\
\frac{\rho}{\alpha} w_{t t}=\beta(u w)_{x x}+\frac{\rho}{\alpha} \frac{l^{2}}{12} w_{x x t t} .
\end{gathered}
$$

Using the wave variable $\xi=x-V t$ and then by integrating this equation twice and considering the constants of integration to be zero, the system (21) is carried to a system of ODEs:

$$
\begin{gathered}
12\left(\alpha \beta-\rho V^{2}\right) U-6 \alpha \beta^{2} U^{2}+6 \alpha \beta W^{2}+\rho l^{2} V^{2} U^{\prime \prime}=0, \\
12 \alpha \beta U W+\rho l^{2} V^{2} W^{\prime \prime}-12 \rho V^{2} W=0 .
\end{gathered}
$$

Balancing $-6 \alpha \beta^{2} U^{2}$ with $\rho l^{2} V^{2} U^{\prime \prime}$ and $12 \alpha \beta U W$ with $\rho l^{2} V^{2} W^{\prime \prime}$ in (22) gives

$$
\begin{aligned}
& 2 M=M+2, \\
& 2 N=M+N,
\end{aligned}
$$

so that $M=N=2$. The tanh-coth method admits the use of

$$
\begin{aligned}
& U(\mu \xi)=S(Y)=\sum_{k=0}^{2} a_{k} Y^{k}+\sum_{k=1}^{2} b_{k} Y^{-k}, \\
& W(\mu \xi)=S(Y)=\sum_{k=0}^{2} c_{k} Y^{k}+\sum_{k=1}^{2} d_{k} Y^{k} .
\end{aligned}
$$

Substituting (24) into (22), collecting the coefficients of $Y$, and solving the resulting system, we find the following sets of solutions:

$$
\begin{gathered}
a_{0}=\frac{1}{\beta+1}, \quad a_{2}=\frac{-1}{2(\beta+1)}, \quad b_{2}=a_{2}, \\
d_{2}= \pm \frac{\sqrt{\beta+2}}{2(\beta+1)}, \quad c_{2}=-d_{2}, \\
V=\frac{\sqrt{3 \rho \alpha \beta(\beta+1)}}{3 \rho(\beta+1)}, \quad \mu=\frac{\sqrt{3}}{l},
\end{gathered}
$$

$$
\begin{gathered}
a_{1}=b_{1}=c_{0}=c_{1}=d_{1}=0, \\
a_{0}=\frac{1}{\beta+1}, \quad a_{2}=\frac{3 \beta}{6 \beta^{2}+14 \beta+8}, \quad b_{2}=a_{2}, \\
d_{2}= \pm \frac{3 \beta \sqrt{\beta+2}}{2(3 \beta+4)(\beta+1)}, \quad c_{2}=-d_{2}, \\
V=\frac{\sqrt{15 \rho \alpha \beta\left(2 l^{2} \mu^{2}+9 \beta+15\right)}}{\rho\left(2 l^{2} \mu^{2}+9 \beta+15\right)}, \\
\mu=\frac{\sqrt{-\beta(15 \beta+12)}}{(5 \beta+4) l}, \quad a_{1}=b_{1}=c_{0}=c_{1}=d_{1}=0, \\
a_{0}=\frac{4-\beta}{4(\beta+1)}, \quad a_{2}=\frac{3 \beta}{4 \beta+1}, \quad c_{0}=\frac{-c_{2}}{3},
\end{gathered}
$$$$
\begin{gathered}
c_{2}= \pm \frac{9 \beta}{2 \sqrt{6 l^{2} \mu^{2}+36 \beta+18}}, \quad V=\frac{\sqrt{-3 \rho \alpha \beta\left(-3+l^{2} \mu^{2}\right)}}{\rho\left(-3+l^{2} \mu^{2}\right)}, \\
\mu=\frac{\sqrt{-\beta(3 \beta+6)}}{(\beta+2) l}, \quad a_{1}=b_{1}=b_{2}=c_{1}=d_{1}=d_{2}=0,
\end{gathered}
$$$$
a_{0}=\frac{3 \beta+4}{4(\beta+1)}, \quad a_{2}=\frac{-3 \beta}{4 \beta+1}, \quad c_{0}=-c_{2},
$$$$
c_{2}= \pm \frac{9 \beta}{2 \sqrt{-6 l^{2} \mu^{2}+36 \beta+18}}, \quad V=\frac{\sqrt{3 \rho \alpha \beta\left(3+l^{2} \mu^{2}\right)}}{\rho\left(3+l^{2} \mu^{2}\right)} \text {, }
$$$$
\mu=\frac{\sqrt{3 \beta(\beta+2)}}{(\beta+2) l}, \quad a_{1}=b_{1}=b_{2}=c_{1}=d_{1}=d_{2}=0,
$$$$
a_{0}=\frac{\beta+8}{8(\beta+1)}, \quad a_{2}=\frac{3 \beta}{16(\beta+1)},
$$$$
b_{2}=a_{2}, \quad c_{0}=\frac{2 d_{2}}{3},
$$$$
c_{2}=d_{2}, \quad d_{2}= \pm \frac{9 \beta}{8 \sqrt{24 l^{2} \mu^{2}+36 \beta+18}},
$$

$$
\begin{gathered}
V=\frac{\sqrt{-3 \rho \alpha \beta\left(-3+4 l^{2} \mu^{2}\right)}}{\rho\left(-3+4 l^{2} \mu^{2}\right)}, \quad \mu=\frac{\sqrt{-\beta(3 \beta+2(\beta+2)}}{a_{1}=b_{1}=c_{1}=d_{1}=0,} \\
a_{0}=\frac{3(\beta+8)}{8(\beta+1)}, \quad a_{2}=\frac{-3 \beta}{16(\beta+1)}, \\
b_{2}=a_{2}, \quad c_{0}=-2 d_{2},
\end{gathered}
$$$$
\mu=\frac{\sqrt{-\beta(3 \beta+6)}}{2(\beta+2) l},
$$ 


$$
\begin{gathered}
c_{2}=d_{2}, \quad d_{2}= \pm \frac{9 \beta}{8 \sqrt{-24 l^{2} \mu^{2}+36 \beta+18}}, \\
V=\frac{\sqrt{3 \rho \alpha \beta\left(3+4 l^{2} \mu^{2}\right)}}{\rho\left(3+4 l^{2} \mu^{2}\right)}, \quad \mu=\frac{\sqrt{\beta(3 \beta+6)}}{2(\beta+2) l}, \\
a_{1}=b_{1}=c_{1}=d_{1}=0 .
\end{gathered}
$$

In accordance with these sets of solutions, we have the following solutions:

$$
\begin{aligned}
u_{1}(x, t)= & \frac{1}{\beta+1}-\frac{1}{2(\beta+1)} \tanh ^{2} \frac{\sqrt{3}}{l} \\
& \times\left(x-\frac{\sqrt{3 \rho \alpha \beta(\beta+1)}}{3 \rho(\beta+1)} t\right) \\
& -\frac{1}{2(\beta+1)} \operatorname{coth}^{2} \frac{\sqrt{3}}{l}\left(x-\frac{\sqrt{3 \rho \alpha \beta(\beta+1)}}{3 \rho(\beta+1)} t\right), \\
w_{1}(x, t)= & \mp \frac{\sqrt{\beta+2}}{2(\beta+1)} \tanh ^{2} \frac{\sqrt{3}}{l}\left(x-\frac{\sqrt{3 \rho \alpha \beta(\beta+1)}}{3 \rho(\beta+1)} t\right) \\
& \pm \frac{\sqrt{\beta+2}}{2(\beta+1)} \operatorname{coth}^{2} \frac{\sqrt{3}}{l}\left(x-\frac{\sqrt{3 \rho \alpha \beta(\beta+1)}}{3 \rho(\beta+1)} t\right),
\end{aligned}
$$$$
u_{2}(x, t)=\frac{1}{\beta+1}
$$

$$
+\frac{3 \beta}{6 \beta^{2}+14 \beta+8} \tanh ^{2} \frac{\sqrt{-\beta(15 \beta+12)}}{(5 \beta+4) l}
$$$$
\times\left(x-\frac{\sqrt{15 \rho \alpha \beta\left(2 l^{2} \mu^{2}+9 \beta+15\right)}}{\rho\left(2 l^{2} \mu^{2}+9 \beta+15\right)} t\right)
$$$$
+\frac{3 \beta}{6 \beta^{2}+14 \beta+8} \operatorname{coth}^{2} \frac{\sqrt{-\beta(15 \beta+12)}}{(5 \beta+4) l}
$$$$
\times\left(x-\frac{\sqrt{15 \rho \alpha \beta\left(2 l^{2} \mu^{2}+9 \beta+15\right)}}{\rho\left(2 l^{2} \mu^{2}+9 \beta+15\right)} t\right),
$$

$w_{2}(x, t)=\mp \frac{3 \beta \sqrt{\beta+2}}{2(3 \beta+4)(\beta+1)} \tanh ^{2} \frac{\sqrt{-\beta(15 \beta+12)}}{(5 \beta+4) l}$

$$
\begin{aligned}
& \times\left(x-\frac{\sqrt{15 \rho \alpha \beta\left(2 l^{2} \mu^{2}+9 \beta+15\right)}}{\rho\left(2 l^{2} \mu^{2}+9 \beta+15\right)} t\right) \\
& \pm \frac{3 \beta \sqrt{\beta+2}}{2(3 \beta+4)(\beta+1)} \operatorname{coth}^{2} \frac{\sqrt{-\beta(15 \beta+12)}}{(5 \beta+4) l}
\end{aligned}
$$$$
\times\left(x-\frac{\sqrt{15 \rho \alpha \beta\left(2 l^{2} \mu^{2}+9 \beta+15\right)}}{\rho\left(2 l^{2} \mu^{2}+9 \beta+15\right)} t\right),
$$

$$
\begin{aligned}
u_{3}(x, t)= & \frac{4-\beta}{4(\beta+1)}+\frac{3 \beta}{4 \beta+1} \tanh ^{2} \frac{\sqrt{-\beta(3 \beta+6)}}{(\beta+2) l} \\
& \times\left(x-\frac{\sqrt{-3 \rho \alpha \beta\left(-3+l^{2} \mu^{2}\right)}}{\rho\left(-3+l^{2} \mu^{2}\right)} t\right),
\end{aligned}
$$

$w_{3}(x, t)=\mp \frac{3 \beta}{2 \sqrt{6 l^{2} \mu^{2}+36 \beta+18}}$

$$
\pm \frac{9 \beta}{2 \sqrt{6 l^{2} \mu^{2}+36 \beta+18}} \tanh ^{2} \frac{\sqrt{-\beta(3 \beta+6)}}{(\beta+2) l}
$$$$
\times\left(x-\frac{\sqrt{-3 \rho \alpha \beta\left(-3+l^{2} \mu^{2}\right)}}{\rho\left(-3+l^{2} \mu^{2}\right)} t\right),
$$

$$
\begin{aligned}
u_{4}(x, t)= & \frac{3 \beta+4}{4(\beta+1)}-\frac{3 \beta}{4 \beta+1} \tanh ^{2} \frac{\sqrt{3 \beta(\beta+2)}}{(\beta+2) l} \\
& \times\left(x-\frac{\sqrt{3 \rho \alpha \beta\left(3+l^{2} \mu^{2}\right)}}{\rho\left(3+l^{2} \mu^{2}\right)} t\right),
\end{aligned}
$$

$$
w_{4}(x, t)=\mp \frac{9 \beta}{2 \sqrt{-6 l^{2} \mu^{2}+36 \beta+18}}
$$

$$
\pm \frac{9 \beta}{2 \sqrt{-6 l^{2} \mu^{2}+36 \beta+18}} \tanh ^{2} \frac{\sqrt{3 \beta(\beta+2)}}{(\beta+2) l}
$$

$$
\times\left(x-\frac{\sqrt{3 \rho \alpha \beta\left(3+l^{2} \mu^{2}\right)}}{\rho\left(3+l^{2} \mu^{2}\right)} t\right),
$$

$u_{5}(x, t)=\frac{\beta+8}{8(\beta+1)}+\frac{3 \beta}{16(\beta+1)} \tanh ^{2} \frac{\sqrt{-\beta(3 \beta+6)}}{2(\beta+2) l}$ 


$$
\begin{aligned}
& \times\left(x-\frac{\sqrt{-3 \rho \alpha \beta\left(-3+4 l^{2} \mu^{2}\right)}}{\rho\left(-3+4 l^{2} \mu^{2}\right)} t\right) \\
& +\frac{3 \beta}{16(\beta+1)} \operatorname{coth}^{2} \frac{\sqrt{-\beta(3 \beta+6)}}{2(\beta+2) l} \\
& \times\left(x-\frac{\sqrt{-3 \rho \alpha \beta\left(-3+4 l^{2} \mu^{2}\right)}}{\rho\left(-3+4 l^{2} \mu^{2}\right)} t\right), \\
& w_{5}(x, t)= \pm \frac{3 \beta}{4 \sqrt{24 l^{2} \mu^{2}+36 \beta+18}} \\
& \pm \frac{9 \beta}{8 \sqrt{24 l^{2} \mu^{2}+36 \beta+18}} \tanh ^{2} \frac{\sqrt{-\beta(3 \beta+6)}}{2(\beta+2) l} \\
& \times\left(x-\frac{\sqrt{-3 \rho \alpha \beta\left(-3+4 l^{2} \mu^{2}\right)}}{\rho\left(-3+4 l^{2} \mu^{2}\right)} t\right) \\
& \pm \frac{9 \beta}{8 \sqrt{24 l^{2} \mu^{2}+36 \beta+18}} \operatorname{coth}^{2} \frac{\sqrt{-\beta(3 \beta+6)}}{2(\beta+2) l} \\
& \times\left(x-\frac{\sqrt{-3 \rho \alpha \beta\left(-3+4 l^{2} \mu^{2}\right)}}{\rho\left(-3+4 l^{2} \mu^{2}\right)} t\right) \\
& u_{6}(x, t)=\frac{3(\beta+8)}{8(\beta+1)}-\frac{3 \beta}{16(\beta+1)} \tanh ^{2} \frac{\sqrt{\beta(3 \beta+6)}}{2(\beta+2) l} \\
& \times\left(x-\frac{\sqrt{3 \rho \alpha \beta\left(3+4 l^{2} \mu^{2}\right)}}{\rho\left(3+4 l^{2} \mu^{2}\right)} t\right) \\
& -\frac{3 \beta}{16(\beta+1)} \operatorname{coth}^{2} \frac{\sqrt{\beta(3 \beta+6)}}{2(\beta+2) l} \\
& \times\left(x-\frac{\sqrt{3 \rho \alpha \beta\left(3+4 l^{2} \mu^{2}\right)}}{\rho\left(3+4 l^{2} \mu^{2}\right)} t\right) \\
& w_{6}(x, t)=\mp \frac{9 \beta}{4 \sqrt{-24 l^{2} \mu^{2}+36 \beta+18}} \\
& \pm \frac{9 \beta}{8 \sqrt{-24 l^{2} \mu^{2}+36 \beta+18}} \tanh ^{2} \frac{\sqrt{\beta(3 \beta+6)}}{2(\beta+2) l}
\end{aligned}
$$

3.3. The Third Coupled. We now examine the coupled equations

$$
\begin{gathered}
u_{t t}=\beta u_{x x}-\frac{\beta}{2}\left(u^{2}\right)_{x x}+\frac{1}{2}\left(w^{2}\right)_{x x}+\alpha^{2} u_{x x t t}, \\
w_{t t}=(u w)_{x x}+\alpha^{2} w_{x x t t} .
\end{gathered}
$$

Using the wave variable $\xi=x-V t$, the system (27) is carried to a system of ODEs. Integrating these equations twice and neglecting constants of integration we find

$$
\begin{gathered}
2\left(V^{2}-\beta\right) U+\beta U^{2}-\beta W^{2}-2 \alpha^{2} V^{2} U^{\prime \prime}=0, \\
V^{2} W-U W-\alpha^{2} V^{2} W^{\prime \prime}=0 .
\end{gathered}
$$

Balancing $\beta W^{2}$ with $2 \alpha^{2} V^{2} U^{\prime \prime}$ and $V^{2} W^{\prime \prime}$ with $U W$ in (28) gives

$$
\begin{gathered}
2 N=M+2, \\
2+N=M+N,
\end{gathered}
$$

so that

$$
M=N=2
$$

As a result, we seek solutions to (27) in the form

$$
\begin{aligned}
& U(\mu \xi)=S(Y)=\sum_{k=0}^{2} a_{k} Y^{k}+\sum_{k=1}^{2} b_{k} Y^{-k} \\
& W(\mu \xi)=S(Y)=\sum_{k=0}^{2} c_{k} Y^{k}+\sum_{k=1}^{2} d_{k} Y^{k}
\end{aligned}
$$

Substitution of (31) into (28) leads to an algebraic system in the unknowns $a_{0}, a_{1}, a_{2}, b_{1}, b_{2}, c_{0}, c_{1}, c_{2}, d_{1}, d_{2}, V$, and $\mu$. 
Collecting the coefficients of $Y$ and solving the resulting system, we find the following sets of solutions:

$$
\begin{aligned}
& a_{0}=\frac{\beta(3 \beta+8)}{8(\beta+1)}, \quad a_{2}=\frac{-3 \beta^{2}}{16(\beta+1)}, \\
& b_{2}=a_{2}, \quad c_{0}=-2 d_{2}, \quad c_{2}=d_{2}, \\
& d_{2}=\frac{ \pm 3 \sqrt{-4 V^{4}-4 V^{2}+2 \beta^{2}+4 \beta}}{16} \\
& V=\frac{\sqrt{\beta\left(16 \alpha^{2} \mu^{2}+1\right)}}{16 \alpha^{2} \mu^{2}+1}, \quad \mu=\frac{\sqrt{\beta(\beta+2)}}{4 \alpha(\beta+2)}, \\
& a_{1}=b_{1}=c_{1}=d_{1}=0 \text {, } \\
& a_{0}=\frac{\beta(\beta+8)}{8(\beta+1)}, \quad a_{2}=\frac{3 \beta^{2}}{16(\beta+1)}, \quad b_{2}=a_{2}, \\
& c_{0}=\frac{2 d_{2}}{3}, \quad c_{2}=d_{2}, \\
& d_{2}=\frac{ \pm 3 \sqrt{-4 V^{4}-4 V^{2}+2 \beta^{2}+4 \beta}}{16} \\
& V=\frac{\sqrt{-\beta\left(16 \alpha^{2} \mu^{2}-1\right)}}{16 \alpha^{2} \mu^{2}-1}, \quad \mu=\frac{\sqrt{-\beta(\beta+2)}}{4 \alpha(\beta+2)}, \\
& a_{1}=b_{1}=c_{1}=d_{1}=0, \\
& a_{0}=\frac{-\beta(\beta-4)}{4(\beta+1)}, \quad a_{2}=\frac{3 \beta^{2}}{4(\beta+1)}, \quad c_{0}=-\frac{c_{2}}{3} \text {, } \\
& c_{2}=\frac{ \pm 3 \sqrt{-4 V^{4}-4 V^{2}+2 \beta^{2}+4 \beta}}{4} \\
& V=\frac{\sqrt{-\beta\left(4 \alpha^{2} \mu^{2}-1\right)}}{4 \alpha^{2} \mu^{2}-1}, \quad \mu=\frac{\sqrt{-\beta(\beta+2)}}{2 \alpha(\beta+2)}, \\
& a_{1}=b_{1}=b_{2}=c_{1}=d_{1}=d_{2}=0, \\
& a_{0}=\frac{\beta(3 \beta+4)}{4(\beta+1)}, \quad a_{2}=\frac{-3 \beta^{2}}{4(\beta+1)}, \quad c_{0}=-2 \text {, } \\
& c_{2}=\frac{ \pm 3 \sqrt{-4 V^{4}-4 V^{2}+2 \beta^{2}+4 \beta}}{4} \\
& V=\frac{\sqrt{\beta\left(4 \alpha^{2} \mu^{2}+1\right)}}{4 \alpha^{2} \mu^{2}+1}, \quad \mu=\frac{\sqrt{\beta(\beta+2)}}{2 \alpha(\beta+2)}, \\
& a_{1}=b_{1}=b_{2}=c_{1}=d_{1}=d_{2}=0, \\
& a_{0}=\frac{\beta}{\beta+1}, \quad a_{2}=\frac{3 \beta^{2}}{2\left(3 \beta^{2}+7 \beta+4\right)},
\end{aligned}
$$

$$
b_{2}=a_{2}, \quad c_{2}=-d_{2}
$$

$$
\begin{gathered}
d_{2}=\frac{ \pm 3 \sqrt{-42 V^{4} \beta-58 V^{4}-2 V^{2} \beta-120 V^{2}+120 \beta}}{8}, \quad \mu=\frac{\sqrt{-\beta(5 \beta+4)}}{2 \alpha(5 \beta+4)}, \\
V=\frac{\sqrt{5 \beta\left(8 \alpha^{2} \mu^{2}+3 \beta+5\right)}}{8 \alpha^{2} \mu^{2}+3 \beta+5}, \quad \mu=c_{1}=c_{1}=d_{1}=0,
\end{gathered}
$$$$
a_{0}=\frac{\beta}{\beta+1}, \quad a_{2}=\frac{-\beta}{2(\beta+1)}, \quad b_{2}=a_{2} \text {, }
$$$$
c_{2}=-d_{2}, \quad d_{2}=\frac{ \pm \sqrt{9 V^{2}+6}}{2},
$$$$
V=\frac{\sqrt{3 \beta(\beta+1)}}{3(\beta+1)}, \quad \mu=\frac{-1}{2 \alpha},
$$$$
a_{1}=b_{1}=c_{0}=c_{1}=d_{1}=0 \text {. }
$$

Consequently, we obtain the following solutions:

$$
\begin{aligned}
& u_{1}(x, t)=\frac{\beta(3 \beta+8)}{8(\beta+1)}-\frac{3 \beta^{2}}{16(\beta+1)} \tanh ^{2} \frac{\sqrt{\beta(\beta+2)}}{4 \alpha(\beta+2)} \\
& \times\left(x-\frac{\sqrt{\beta\left(16 \alpha^{2} \mu^{2}+1\right)}}{16 \alpha^{2} \mu^{2}+1} t\right) \\
& -\frac{3 \beta^{2}}{16(\beta+1)} \operatorname{coth}^{2} \frac{\sqrt{\beta(\beta+2)}}{4 \alpha(\beta+2)} \\
& \times\left(x-\frac{\sqrt{\beta\left(16 \alpha^{2} \mu^{2}+1\right)}}{16 \alpha^{2} \mu^{2}+1} t\right) \text {, } \\
& w_{1}(x, t)=\frac{\mp 3 \sqrt{-4 V^{4}-4 V^{2}+2 \beta^{2}+4 \beta}}{8} \\
& \pm \frac{3 \sqrt{-4 V^{4}-4 V^{2}+2 \beta^{2}+4 \beta}}{16} \tanh ^{2} \frac{\sqrt{\beta(\beta+2)}}{4 \alpha(\beta+2)} \\
& \times\left(x-\frac{\sqrt{\beta\left(16 \alpha^{2} \mu^{2}+1\right)}}{16 \alpha^{2} \mu^{2}+1} t\right) \\
& \pm \frac{3 \sqrt{-4 V^{4}-4 V^{2}+2 \beta^{2}+4 \beta}}{16} \operatorname{coth}^{2} \frac{\sqrt{\beta(\beta+2)}}{4 \alpha(\beta+2)} \\
& \times\left(x-\frac{\sqrt{\beta\left(16 \alpha^{2} \mu^{2}+1\right)}}{16 \alpha^{2} \mu^{2}+1} t\right)
\end{aligned}
$$




$$
\begin{aligned}
& u_{2}(x, t)=\frac{\beta(\beta+8)}{8(\beta+1)}+\frac{3 \beta^{2}}{16(\beta+1)} \tanh ^{2} \frac{\sqrt{-\beta(\beta+2)}}{4 \alpha(\beta+2)} \\
& u_{4}(x, t)=\frac{\beta(3 \beta+4)}{4(\beta+1)}-\frac{3 \beta^{2}}{4(\beta+1)} \tanh ^{2} \frac{\sqrt{\beta(\beta+2)}}{2 \alpha(\beta+2)} \\
& \times\left(x-\frac{\sqrt{-\beta\left(16 \alpha^{2} \mu^{2}-1\right)}}{16 \alpha^{2} \mu^{2}-1} t\right) \\
& \times\left(x-\frac{\sqrt{\beta\left(4 \alpha^{2} \mu^{2}+1\right)}}{4 \alpha^{2} \mu^{2}+1} t\right) \\
& +\frac{3 \beta^{2}}{16(\beta+1)} \operatorname{coth}^{2} \frac{\sqrt{-\beta(\beta+2)}}{4 \alpha(\beta+2)} \\
& \times\left(x-\frac{\sqrt{-\beta\left(16 \alpha^{2} \mu^{2}-1\right)}}{16 \alpha^{2} \mu^{2}-1} t\right), \\
& w_{2}(x, t)= \pm \frac{\sqrt{-4 V^{4}-4 V^{2}+2 \beta^{2}+4 \beta}}{8} \\
& \pm \frac{3 \sqrt{-4 V^{4}-4 V^{2}+2 \beta^{2}+4 \beta}}{16} \tanh ^{2} \frac{\sqrt{-\beta(\beta+2)}}{4 \alpha(\beta+2)} \\
& \times\left(x-\frac{\sqrt{-\beta\left(16 \alpha^{2} \mu^{2}-1\right)}}{16 \alpha^{2} \mu^{2}-1} t\right) \\
& \pm \frac{3 \sqrt{-4 V^{4}-4 V^{2}+2 \beta^{2}+4 \beta}}{16} \operatorname{coth}^{2} \frac{\sqrt{-\beta(\beta+2)}}{4 \alpha(\beta+2)} \\
& \times\left(x-\frac{\sqrt{-\beta\left(16 \alpha^{2} \mu^{2}-1\right)}}{16 \alpha^{2} \mu^{2}-1} t\right) \\
& u_{3}(x, t)=\frac{-\beta(\beta-4)}{4(\beta+1)}+\frac{3 \beta^{2}}{4(\beta+1)} \tanh ^{2} \frac{\sqrt{-\beta(\beta+2)}}{2 \alpha(\beta+2)} \\
& \times\left(x-\frac{\sqrt{-\beta\left(4 \alpha^{2} \mu^{2}-1\right)}}{4 \alpha^{2} \mu^{2}-1} t\right), \\
& w_{3}(x, t)=\mp \frac{\sqrt{-4 V^{4}-4 V^{2}+2 \beta^{2}+4 \beta}}{4} \\
& \pm \frac{3 \sqrt{-4 V^{4}-4 V^{2}+2 \beta^{2}+4 \beta}}{4} \tanh \frac{\sqrt{-\beta(\beta+2)}}{2 \alpha(\beta+2)} \\
& \times\left(x-\frac{\sqrt{-\beta\left(4 \alpha^{2} \mu^{2}-1\right)}}{4 \alpha^{2} \mu^{2}-1} t\right) \\
& \begin{aligned}
w_{4}(x, t)= & \mp \frac{3 \sqrt{-4 V^{4}-4 V^{2}+2 \beta^{2}+4 \beta}}{4} \\
& \pm \frac{3 \sqrt{-4 V^{4}-4 V^{2}+2 \beta^{2}+4 \beta}}{4} \tanh ^{2} \frac{\sqrt{\beta(\beta+2)}}{2 \alpha(\beta+2)} \\
& \times\left(x-\frac{\sqrt{\beta\left(4 \alpha^{2} \mu^{2}+1\right)}}{4 \alpha^{2} \mu^{2}+1} t\right)
\end{aligned} \\
& u_{5}(x, t)=\frac{\beta}{\beta+1} \\
& +\frac{3 \beta^{2}}{2\left(3 \beta^{2}+7 \beta+4\right)} \tanh ^{2} \frac{\sqrt{-\beta(5 \beta+4)}}{2 \alpha(5 \beta+4)} \\
& \times\left(x-\frac{\sqrt{5 \beta\left(8 \alpha^{2} \mu^{2}+3 \beta+5\right)}}{8 \alpha^{2} \mu^{2}+3 \beta+5} t\right) \\
& +\frac{3 \beta^{2}}{2\left(3 \beta^{2}+7 \beta+4\right)} \operatorname{coth}^{2} \frac{\sqrt{-\beta(5 \beta+4)}}{2 \alpha(5 \beta+4)} \\
& \times\left(x-\frac{\sqrt{5 \beta\left(8 \alpha^{2} \mu^{2}+3 \beta+5\right)}}{8 \alpha^{2} \mu^{2}+3 \beta+5} t\right) \\
& w_{5}(x, t) \\
& =\mp \frac{3 \sqrt{-42 V^{4} \beta-58 V^{4}-2 V^{2} \beta-120 V^{2}+120 \beta}}{8} \\
& \times \tanh ^{2} \frac{\sqrt{-\beta(5 \beta+4)}}{2 \alpha(5 \beta+4)} \\
& \times\left(x-\frac{\sqrt{5 \beta\left(8 \alpha^{2} \mu^{2}+3 \beta+5\right)}}{8 \alpha^{2} \mu^{2}+3 \beta+5} t\right) \\
& \pm \frac{3 \sqrt{-42 V^{4} \beta-58 V^{4}-2 V^{2} \beta-120 V^{2}+120 \beta}}{8}
\end{aligned}
$$




$$
\left.\begin{array}{rl} 
& \times \operatorname{coth}^{2} \frac{\sqrt{-\beta(5 \beta+4)}}{2 \alpha(5 \beta+4)} \\
& \times\left(x-\frac{\sqrt{5 \beta\left(8 \alpha^{2} \mu^{2}+3 \beta+5\right)}}{8 \alpha^{2} \mu^{2}+3 \beta+5} t\right) \\
u_{6}(x, t)= & \frac{\beta}{\beta+1} \\
& -\frac{\beta}{2(\beta+1)} \tanh ^{2} \frac{-1}{2 \alpha}\left(x-\frac{\sqrt{3 \beta(\beta+1)}}{3(\beta+1)} t\right) \\
& -\frac{\beta}{2(\beta+1)} \operatorname{coth}^{2} \frac{-1}{2 \alpha}\left(x-\frac{\sqrt{3 \beta(\beta+1)}}{3(\beta+1)} t\right) \\
& \pm \frac{\sqrt{9 V^{2}+6}}{2} \operatorname{coth}^{2} \frac{-1}{2 \alpha}\left(x-\frac{\sqrt{3 \beta(\beta+1)}}{3(\beta+1)} t\right.
\end{array}\right) .
$$

3.4. The Fourth Coupled. We now consider the coupled IBq equations

$$
\begin{gathered}
u_{t t}=u_{x x}-\beta\left(u^{2}\right)_{x x}+\left(w^{2}\right)_{x x}+u_{x x t t}, \\
w_{t t}=w_{x x}+2(u w)_{x x}+w_{x x t t} .
\end{gathered}
$$

Using the wave variable $\xi=x-V t$, (34) can be converted to a system of ODEs:

$$
\begin{gathered}
\left(V^{2}-1\right) U+\beta U^{2}-W^{2}-V^{2} U^{\prime \prime}=0 \\
\left(V^{2}-1\right) W-2 U W-V^{2} W^{\prime \prime}=0 .
\end{gathered}
$$

Balancing $W^{2}$ with $U^{\prime \prime}$ and $U W$ with $W^{\prime \prime}$ in (35) gives

$$
\begin{gathered}
2 N=M+2, \\
M+N=N+2,
\end{gathered}
$$

so that

$$
M=N=2 .
$$

The tanh-coth method substitutes the finite expansions

$$
\begin{aligned}
& U(\mu \xi)=S(Y)=\sum_{k=0}^{2} a_{k} Y^{k}+\sum_{k=1}^{2} b_{k} Y^{-k}, \\
& W(\mu \xi)=S(Y)=\sum_{k=0}^{2} c_{k} Y^{k}+\sum_{k=1}^{2} d_{k} Y^{k} .
\end{aligned}
$$

In (35), collecting the coefficients of each power of $Y$ and solving the resulting system, we obtain

$$
\begin{gathered}
a_{0}=\frac{-2 \mu^{2}}{16 \mu^{2}+1}, \quad a_{2}=\frac{-3 \mu^{2}}{16 \mu^{2}+1}, \quad a_{2}=b_{2}, \\
c_{0}=\frac{2 d_{2}}{3}, \quad c_{2}=d_{2}, \\
d_{2}=\frac{ \pm 3(V+1)(V-1) \sqrt{\beta+2}}{16}, \quad V=\frac{1}{\sqrt{16 \mu^{2}+1}}, \\
a_{0}=\frac{-6 \mu^{2}}{16 \mu^{2}-1}, \quad b_{1}=c_{1}=d_{1}=0, \\
c_{0}=-2 d_{2}, \quad c_{2}=d_{2}, \\
d_{2}=\frac{ \pm 3(V+1)(V-1) \sqrt{\beta+2}}{16}, \quad V=\frac{1}{\sqrt{-16 \mu^{2}+1}},
\end{gathered}
$$$$
a_{1}=b_{1}=c_{1}=d_{1}=0
$$

$$
\begin{gathered}
a_{0}=\frac{\mu^{2}}{4 \mu^{2}+1}, \quad a_{2}=\frac{-3 \mu^{2}}{4 \mu^{2}+1}, \quad c_{0}=\frac{-c_{2}}{3}, \\
c_{2}=\frac{ \pm 3(V+1)(V-1) \sqrt{\beta+2}}{4}, \quad V=\frac{1}{\sqrt{4 \mu^{2}+1}},
\end{gathered}
$$

$$
\begin{gathered}
a_{1}=b_{1}=b_{2}=c_{1}=d_{1}=d_{2}=0, \\
a_{0}=\frac{-3 \mu^{2}}{4 \mu^{2}-1}, \quad a_{2}=\frac{3 \mu^{2}}{4 \mu^{2}-1}, \quad c_{0}=-c_{2}, \\
c_{2}=\frac{ \pm 3(V+1)(V-1) \sqrt{\beta+2}}{4}, \quad V=\frac{1}{\sqrt{-4 \mu^{2}+1}},
\end{gathered}
$$

$$
a_{1}=b_{1}=b_{2}=c_{1}=d_{1}=d_{2}=0,
$$

$$
\begin{gathered}
a_{0}=\frac{-3 \mu^{2}}{4 \mu^{2}-1}, \quad b_{2}=\frac{3 \mu^{2}}{4 \mu^{2}-1}, \quad c_{0}=-d_{2}, \\
d_{2}=\frac{ \pm 3(V+1)(V-1) \sqrt{\beta+2}}{4}, \quad V=\frac{1}{\sqrt{-4 \mu^{2}+1}},
\end{gathered}
$$

$$
a_{1}=a_{2}=b_{1}=c_{1}=c_{2}=d_{1}=0,
$$


where $\mu$ is left as a free parameter. Consequently, we obtain the following travelling wave solutions:

$$
\begin{aligned}
& u_{1}(x, t)=-\frac{2 \mu^{2}}{16 \mu^{2}+1} \\
& -\frac{3 \mu^{2}}{16 \mu^{2}+1} \tanh ^{2} \mu\left(x-\frac{1}{\sqrt{16 \mu^{2}+1}} t\right) \\
& -\frac{3 \mu^{2}}{16 \mu^{2}+1} \operatorname{coth}^{2} \mu\left(x-\frac{1}{\sqrt{16 \mu^{2}+1}} t\right) \text {, } \\
& w_{1}(x, t)= \pm \frac{(V+1)(V-1) \sqrt{\beta+2}}{8} \\
& \pm \frac{3(V+1)(V-1) \sqrt{\beta+2}}{16} \tanh ^{2} \mu \\
& \times\left(x-\frac{1}{\sqrt{16 \mu^{2}+1}} t\right) \\
& \pm \frac{3(V+1)(V-1) \sqrt{\beta+2}}{16} \operatorname{coth}^{2} \mu \\
& \times\left(x-\frac{1}{\sqrt{16 \mu^{2}+1}} t\right), \\
& u_{2}(x, t)=-\frac{6 \mu^{2}}{16 \mu^{2}-1} \\
& +\frac{3 \mu^{2}}{16 \mu^{2}-1} \tanh ^{2} \mu\left(x-\frac{1}{\sqrt{-16 \mu^{2}+1}} t\right) \\
& +\frac{3 \mu^{2}}{16 \mu^{2}-1} \operatorname{coth}^{2} \mu\left(x-\frac{1}{\sqrt{-16 \mu^{2}+1}} t\right) \text {, } \\
& w_{2}(x, t)=\mp \frac{3(V+1)(V-1) \sqrt{\beta+2}}{8} \\
& \pm \frac{3(V+1)(V-1) \sqrt{\beta+2}}{16} \tanh ^{2} \mu \\
& \times\left(x-\frac{1}{\sqrt{-16 \mu^{2}+1}} t\right) \\
& \pm \frac{3(V+1)(V-1) \sqrt{\beta+2}}{16} \operatorname{coth}^{2} \mu \\
& \times\left(x-\frac{1}{\sqrt{-16 \mu^{2}+1}} t\right),
\end{aligned}
$$$$
u_{3}(x, t)=\frac{\mu^{2}}{4 \mu^{2}+1}
$$$$
-\frac{3 \mu^{2}}{4 \mu^{2}+1} \tanh ^{2} \mu\left(x-\frac{1}{\sqrt{4 \mu^{2}+1}} t\right),
$$$$
w_{3}(x, t)=\mp \frac{(V+1)(V-1) \sqrt{\beta+2}}{4}
$$$$
\pm \frac{3(V+1)(V-1) \sqrt{\beta+2}}{4}
$$$$
\times \tanh ^{2} \mu\left(x-\frac{1}{\sqrt{4 \mu^{2}+1}} t\right)
$$$$
u_{4}(x, t)=-\frac{3 \mu^{2}}{4 \mu^{2}-1}
$$$$
+\frac{3 \mu^{2}}{4 \mu^{2}-1} \tanh ^{2} \mu\left(x-\frac{1}{\sqrt{-4 \mu^{2}+1}} t\right),
$$$$
w_{4}(x, t)=\mp \frac{3(V+1)(V-1) \sqrt{\beta+2}}{4}
$$$$
\pm \frac{3(V+1)(V-1) \sqrt{\beta+2}}{4}
$$$$
\times \tanh ^{2} \mu\left(x-\frac{1}{\sqrt{-4 \mu^{2}+1}} t\right)
$$$$
u_{5}(x, t)=-\frac{3 \mu^{2}}{4 \mu^{2}-1}+\frac{3 \mu^{2}}{4 \mu^{2}-1} \operatorname{coth}^{2} \mu\left(x-\frac{1}{\sqrt{-4 \mu^{2}+1}} t\right) \text {, }
$$$$
w_{5}(x, t)=\mp \frac{3(V+1)(V-1) \sqrt{\beta+2}}{4}
$$$$
\pm 3 \frac{(V+1)(V-1) \sqrt{\beta+2}}{4}
$$$$
\times \operatorname{coth}^{2} \mu\left(x-\frac{1}{\sqrt{-4 \mu^{2}+1}} t\right)
$$

3.5. The Fifth Coupled. We finally consider the coupled IBq equations

$$
\begin{gathered}
u_{t t}=\beta u_{x x}-\frac{\beta}{2}\left(u^{2}\right)_{x x}+\frac{\beta}{2}\left(w^{2}\right)_{x x}+\alpha^{2} u_{x x t t}, \\
w_{t t}=\gamma(u w)_{x x}+\rho\left(w^{2}\right)_{x x}+\alpha^{2} w_{x x t t} .
\end{gathered}
$$


This system of equations (41) can be converted to the following system of ODEs by using the wave variable $\xi=x-V t$ :

$$
\begin{gathered}
\left(2 V^{2}-2 \beta\right) U+\beta U^{2}-\beta W^{2}-2 \alpha^{2} V^{2} U^{\prime \prime}=0, \\
V^{2} W-\gamma U W-\rho W^{2}-\alpha^{2} V^{2} W^{\prime \prime}=0 .
\end{gathered}
$$

Using the balancing procedure in (42) gives

$$
\begin{aligned}
& 2 M=M+N, \\
& 2 N=M+N,
\end{aligned}
$$

so that

$$
M=N=2 .
$$

We consider solutions in the following form:

$$
\begin{aligned}
& U(\mu \xi)=S(Y)=\sum_{k=0}^{2} a_{k} Y^{k}+\sum_{k=1}^{2} b_{k} Y^{-k} \\
& W(\mu \xi)=S(Y)=\sum_{k=0}^{2} c_{k} Y^{k}+\sum_{k=1}^{2} d_{k} Y^{k}
\end{aligned}
$$

Substituting (45) into (42), collecting the coefficients of $Y$, and solving the resulting system, we find the following sets of solutions:

$$
\begin{aligned}
& a_{0}=(-\beta\left(8 \rho c_{2} \beta^{2} \gamma-8 \beta \gamma^{2} \rho c_{2}-18 \rho^{2} \beta^{2}+24 \beta \rho^{3} c_{2}\right. \\
&\left.\left.+12 \beta^{2} \gamma^{2}-16 \gamma^{3} \rho c_{2}+24 \rho^{3} c_{2} \gamma-3 \gamma \beta^{3}\right)\right) \\
& \times(4(-8 \beta \rho^{3} c_{2} \gamma-4 \rho^{3} \gamma^{2} c_{2}-4 \beta^{2} \rho^{3} c_{2}+3 \rho^{2} \beta^{3} \\
&+ 3 \rho^{2} \gamma \beta^{2}+4 \rho \gamma^{4} c_{2}+4 \beta^{2} \gamma^{2} \rho c_{2} \\
&+\left.\left.8 \beta \gamma^{3} \rho c_{2}-3 \beta^{3} \gamma^{2}-3 \gamma^{3} \beta^{2}\right)\right)^{-1} \\
& c_{0}=(-\left(4 \rho c_{2} \beta+4 c_{2} \rho \gamma-3 \beta^{2}\right) \\
& a_{2} \frac{4 \gamma(\mu+\beta)}{\rho^{2}} \beta^{2} c_{2}+\gamma^{2} c_{2} \beta^{2}+\beta c_{2} \gamma^{3}+2 \gamma^{2} \rho^{2} c_{2} \\
&\left.\left.+3 \gamma \rho^{2} \beta c_{2}-\frac{3}{2} \rho \beta^{2} \gamma-\frac{3}{4} \rho \beta^{3}\right)\right) \\
& \times( 8 \beta \rho^{3} c_{2} \gamma-4 \rho^{3} \gamma^{2} c_{2}-4 \beta^{2} \rho^{3} c_{2}+3 \rho^{2} \beta^{3} \\
&+ 3 \rho^{2} \gamma \beta^{2}+4 \rho \gamma^{4} c_{2}+4 \beta^{2} \gamma^{2} \rho c_{2} \\
&\left.+8 \beta \gamma^{3} \rho c_{2}-3 \beta^{3} \gamma^{2}-3 \gamma^{3} \beta^{2}\right)^{-1}
\end{aligned}
$$$$
c_{2}=\left( \pm 3\left(-\beta \rho+\left(-\rho^{2} \beta^{2}+4 \gamma^{3} \beta-4 \gamma^{2} V^{4}\right.\right.\right.
$$$$
-4 \gamma^{3} V^{2}+2 \beta^{2} \gamma^{2}
$$$$
\left.\left.\left.-4 \rho^{2} \gamma \beta+4 \rho^{2} V^{4}+4 \rho^{2} V^{2} \gamma\right)^{1 / 2}\right)\right)
$$$$
\times\left(4\left(\gamma^{2}-\rho^{2}\right)\right)^{-1}
$$$$
V=\frac{\sqrt{-\beta\left(4 \alpha^{2} \mu^{2}-1\right)}}{4 \alpha^{2} \mu^{2}-1}, \quad \mu=\frac{\sqrt{-\beta(2 \gamma+\beta)}}{2 \alpha(2 \gamma+\beta)},
$$$$
a_{1}=b_{1}=b_{2}=c_{1}=d_{1}=d_{2}=0,
$$

$$
a_{0}=\left(\beta \left(6 \rho c_{2} \beta^{2} \gamma+10 \beta \gamma^{2} \rho c_{2}+\frac{3}{2} \rho^{2} \beta^{2}+2 \beta \rho^{3} c_{2}\right.\right.
$$$$
\left.\left.+3 \beta^{2} \gamma^{2}+4 \gamma^{3} \rho c_{2}+2 \rho^{3} c_{2} \gamma+\frac{9}{4} \gamma \beta^{3}\right)\right)
$$

$$
\times\left(-8 \beta \rho^{3} c_{2} \gamma-4 \rho^{3} \gamma^{2} c_{2}-4 \beta^{2} \rho^{3} c_{2}-3 \rho^{2} \beta^{3}\right.
$$

$$
-3 \rho^{2} \gamma \beta^{2}+4 \rho \gamma^{4} c_{2}+4 \beta^{2} \gamma^{2} \rho c_{2}
$$$$
\left.+8 \beta \gamma^{3} \rho c_{2}+3 \beta^{3} \gamma^{2}+3 \gamma^{3} \beta^{2}\right)^{-1} \text {, }
$$$$
a_{2}=\frac{-\left(4 \rho c_{2} \beta+4 c_{2} \rho \gamma+3 \beta^{2}\right)}{4 \gamma(\mu+\beta)},
$$

$c_{0}=\left(\beta\left(-3 \rho^{2} \beta^{2} c_{2}-3 \gamma^{2} c_{2} \beta^{2}-3 \beta c_{2} \gamma^{3}\right.\right.$

$$
\left.\left.-6 \gamma^{2} \rho^{2} c_{2}-9 \gamma \rho^{2} \beta c_{2}-\frac{9}{2} \rho \beta^{2} \gamma-\frac{9}{4} \rho \beta^{3}\right)\right)
$$

$$
\times\left(-8 \beta \rho^{3} c_{2} \gamma-4 \rho^{3} \gamma^{2} c_{2}-4 \beta^{2} \rho^{3} c_{2}\right.
$$$$
-3 \rho^{2} \beta^{3}-3 \rho^{2} \gamma \beta^{2}+4 \rho \gamma^{4} c_{2}+4 \beta^{2} \gamma^{2} \rho c_{2}
$$$$
\left.+8 \beta \gamma^{3} \rho c_{2}+3 \beta^{3} \gamma^{2}+3 \gamma^{3} \beta^{2}\right)^{-1},
$$

$$
\begin{aligned}
c_{2}=( \pm 3(\beta \rho+( & -\rho^{2} \beta^{2}+4 \gamma^{3} \beta-4 \gamma^{2} V^{4} \\
& -4 \gamma^{3} V^{2}+2 \beta^{2} \gamma^{2}-4 \rho^{2} \gamma \beta \\
& \left.\left.\left.+4 \rho^{2} V^{4}+4 \rho^{2} V^{2} \gamma\right)^{1 / 2}\right)\right)
\end{aligned}
$$

$$
\begin{gathered}
\times\left(4\left(\gamma^{2}-\rho^{2}\right)\right)^{-1}, \\
V=\frac{\sqrt{\beta\left(4 \alpha^{2} \mu^{2}+1\right)}}{4 \alpha^{2} \mu^{2}+1}, \quad \mu=\frac{\sqrt{\beta(2 \gamma+\beta)}}{2 \alpha(2 \gamma+\beta)}, \\
a_{1}=b_{1}=b_{2}=c_{1}=d_{1}=d_{2}=0 .
\end{gathered}
$$


In view of this, we obtain the following travelling wave solutions:

$$
\begin{aligned}
& u_{1}(x, t)=\left(-\beta\left(8 \rho c_{2} \beta^{2} \gamma-8 \beta \gamma^{2} \rho c_{2}-18 \rho^{2} \beta^{2}+24 \beta \rho^{3} c_{2}\right.\right. \\
& \left.\left.+12 \beta^{2} \gamma^{2}-16 \gamma^{3} \rho c_{2}+24 \rho^{3} c_{2} \gamma-3 \gamma \beta^{3}\right)\right) \\
& \times\left(4 \left(-8 \beta \rho^{3} c_{2} \gamma-4 \rho^{3} \gamma^{2} c_{2}-4 \beta^{2} \rho^{3} c_{2}+3 \rho^{2} \beta^{3}\right.\right. \\
& +3 \rho^{2} \gamma \beta^{2}+4 \rho \gamma^{4} c_{2}+4 \beta^{2} \gamma^{2} \rho c_{2} \\
& \left.\left.+8 \beta \gamma^{3} \rho c_{2}-3 \beta^{3} \gamma^{2}-3 \gamma^{3} \beta^{2}\right)\right)^{-1} \\
& -\frac{\left(4 \rho c_{2} \beta+4 c_{2} \rho \gamma-3 \beta^{2}\right)}{4 \gamma(\mu+\beta)} \tanh ^{2} \frac{\sqrt{-\beta(2 \gamma+\beta)}}{2 \alpha(2 \gamma+\beta)} \\
& \times\left(x-\frac{\sqrt{-\beta\left(4 \alpha^{2} \mu^{2}-1\right)}}{4 \alpha^{2} \mu^{2}-1} t\right), \\
& w_{1}(x, t)=\left(\beta \left(\rho^{2} \beta^{2} c_{2}+\gamma^{2} c_{2} \beta^{2}+\beta c_{2} \gamma^{3}+2 \gamma^{2} \rho^{2} c_{2}\right.\right. \\
& \left.\left.+3 \gamma \rho^{2} \beta c_{2}-\frac{3}{2} \rho \beta^{2} \gamma-\frac{3}{4} \rho \beta^{3}\right)\right) \\
& \times\left(-8 \beta \rho^{3} c_{2} \gamma-4 \rho^{3} \gamma^{2} c_{2}-4 \beta^{2} \rho^{3} c_{2}+3 \rho^{2} \beta^{3}\right. \\
& +3 \rho^{2} \gamma \beta^{2}+4 \rho \gamma^{4} c_{2}+4 \beta^{2} \gamma^{2} \rho c_{2} \\
& \left.+8 \beta \gamma^{3} \rho c_{2}-3 \beta^{3} \gamma^{2}-3 \gamma^{3} \beta^{2}\right)^{-1} \\
& \pm\left(3 \left(-\beta \rho+\left(-\rho^{2} \beta^{2}+4 \gamma^{3} \beta-4 \gamma^{2} V^{4}\right.\right.\right. \\
& -4 \gamma^{3} V^{2}+2 \beta^{2} \gamma^{2}-4 \rho^{2} \gamma \beta \\
& \left.\left.\left.+4 \rho^{2} V^{4}+4 \rho^{2} V^{2} \gamma\right)^{1 / 2}\right)\right) \\
& \times\left(4\left(\gamma^{2}-\rho^{2}\right)\right)^{-1} \\
& \times \tanh ^{2} \frac{\sqrt{-\beta(2 \gamma+\beta)}}{2 \alpha(2 \gamma+\beta)} \\
& \times\left(x-\frac{\sqrt{-\beta\left(4 \alpha^{2} \mu^{2}-1\right)}}{4 \alpha^{2} \mu^{2}-1} t\right), \\
& u_{2}(x, t)=\left(\beta \left(6 \rho c_{2} \beta^{2} \gamma+10 \beta \gamma^{2} \rho c_{2}+\frac{3}{2} \rho^{2} \beta^{2}+2 \beta \rho^{3} c_{2}\right.\right. \\
& \left.\left.+3 \beta^{2} \gamma^{2}+4 \gamma^{3} \rho c_{2}+2 \rho^{3} c_{2} \gamma+\frac{9}{4} \gamma \beta^{3}\right)\right) \\
& \times\left(-8 \beta \rho^{3} c_{2} \gamma-4 \rho^{3} \gamma^{2} c_{2}-4 \beta^{2} \rho^{3} c_{2}-3 \rho^{2} \beta^{3}\right. \\
& -3 \rho^{2} \gamma \beta^{2}+4 \rho \gamma^{4} c_{2}+4 \beta^{2} \gamma^{2} \rho c_{2} \\
& \left.+8 \beta \gamma^{3} \rho c_{2}+3 \beta^{3} \gamma^{2}+3 \gamma^{3} \beta^{2}\right)^{-1}
\end{aligned}
$$

$$
\begin{aligned}
- & \frac{\left(4 \rho c_{2} \beta+4 c_{2} \rho \gamma+3 \beta^{2}\right)}{4 \gamma(\mu+\beta)} \tanh ^{2} \frac{\sqrt{\beta(2 \gamma+\beta)}}{2 \alpha(2 \gamma+\beta)} \\
\times & \left(x-\frac{\sqrt{\beta\left(4 \alpha^{2} \mu^{2}+1\right)}}{4 \alpha^{2} \mu^{2}+1} t\right), \\
w_{2}(x, t)= & \left(\beta \left(-3 \rho^{2} \beta^{2} c_{2}-3 \gamma^{2} c_{2} \beta^{2}-3 \beta c_{2} \gamma^{3}\right.\right. \\
& \left.\left.-6 \gamma^{2} \rho^{2} c_{2}-9 \gamma \rho^{2} \beta c_{2}-\frac{9}{2} \rho \beta^{2} \gamma-\frac{9}{4} \rho \beta^{3}\right)\right) \\
\times & \left(-8 \beta \rho^{3} c_{2} \gamma-4 \rho^{3} \gamma^{2} c_{2}-4 \beta^{2} \rho^{3} c_{2}-3 \rho^{2} \beta^{3}\right. \\
& -3 \rho^{2} \gamma \beta^{2}+4 \rho \gamma^{4} c_{2}+4 \beta^{2} \gamma^{2} \rho c_{2} \\
& \left.+8 \beta \gamma^{3} \rho c_{2}+3 \beta^{3} \gamma^{2}+3 \gamma^{3} \beta^{2}\right)^{-1} \\
\pm & \left(3 \left(\beta \rho+\left(-\rho^{2} \beta^{2}+4 \gamma^{3} \beta-4 \gamma^{2} V^{4}\right.\right.\right. \\
& -4 \gamma^{3} V^{2}+2 \beta^{2} \gamma^{2}-4 \rho^{2} \gamma \beta \\
& \left.\left.\left.+4 \rho^{2} V^{4}+4 \rho^{2} V^{2} \gamma\right)^{1 / 2}\right)\right) \\
\times & +\tanh ^{2} \frac{\sqrt{\beta(2 \gamma+\beta)}}{2 \alpha(2 \gamma+\beta)}\left(x-\frac{\sqrt{\beta\left(4 \alpha^{2} \mu^{2}+1\right)}}{4 \alpha^{2} \mu^{2}+1} t\right) . \\
\times & \left(4\left(\gamma^{2}-\rho^{2}\right)\right)^{-1}
\end{aligned}
$$

\section{Conclusion}

In summary, the tanh-coth method has been successfully implemented to find new travelling wave solutions for several kinds of two coupled improved Boussinesq equations. The solutions that have been found can be seen as an expansion and verification of the previously known data. However, this study confirmed again the belief that the tanh-coth method is a powerful and reliable technique to handle nonlinear dispersive-dissipative equations and system of equations. Throughout the work, Maple has been used to overcome the tedious algebraic calculations.

\section{Conflict of Interests}

The authors declare that there is no conflict of interests regarding the publication of this paper.

\section{References}

[1] A. Dé Godefroy, "Blow up of solutions of a generalized Boussinesq equation," IMA Journal of Applied Mathematics, vol. 60, no. 2, pp. 123-138, 1998. 
[2] S. Wang and M. Li, "The Cauchy problem for coupled IMBq equations," IMA Journal of Applied Mathematics, vol. 74, no. 5, pp. 726-740, 2009.

[3] N. Duruk, H. A. Erbay, and A. Erkip, "Blow-up and global existence for a general class of nonlocal nonlinear coupled wave equations," Journal of Differential Equations, vol. 250, no. 3, pp. 1448-1459, 2011.

[4] G. Chen and H. Zhang, "Initial boundary value problem for a system of generalized IMBq equations," Mathematical Methods in the Applied Sciences, vol. 27, no. 5, pp. 497-518, 2004.

[5] P. Rosenau, "Dynamics of dense lattices," Physical Review B, vol. 36, no. 11, pp. 5868-5876, 1987.

[6] P. L. Christiansen, P. S. Lomdahl, and V. Muto, "On a Toda lattice model with a transversal degree of freedom," Nonlinearity, vol. 4, no. 2, pp. 477-501, 1991.

[7] S. K. Turitsyn, "On a Toda lattice model with a transversal degree of freedom. Sufficient criterion of blow-up in the continuum limit," Physics Letters A, vol. 173, no. 3, pp. 267-269, 1993.

[8] R. L. Pego, P. Smereka, and M. I. Weinstein, "Oscillatory instability of solitary waves in a continuum model of lattice vibrations," Nonlinearity, vol. 8, no. 6, pp. 921-941, 1995.

[9] X. Fan and L. Tian, "Compacton solutions and multiple compacton solutions for a continuum Toda lattice model," Chaos, Solitons and Fractals, vol. 29, no. 4, pp. 882-894, 2006.

[10] A. M. Wazwaz, "The Hirota's bilinear method and the tanhcoth method for multiple-soliton solutions of the SawadaKotera-Kadomtsev-Petviashvili equation," Applied Mathematics and Computation, vol. 200, no. 1, pp. 160-166, 2008. 


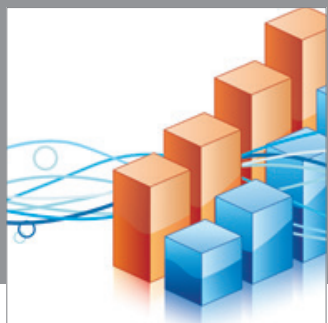

Advances in

Operations Research

mansans

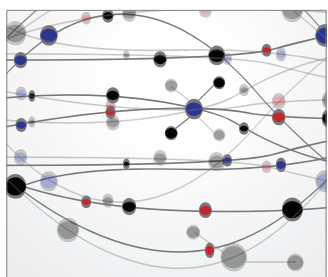

The Scientific World Journal
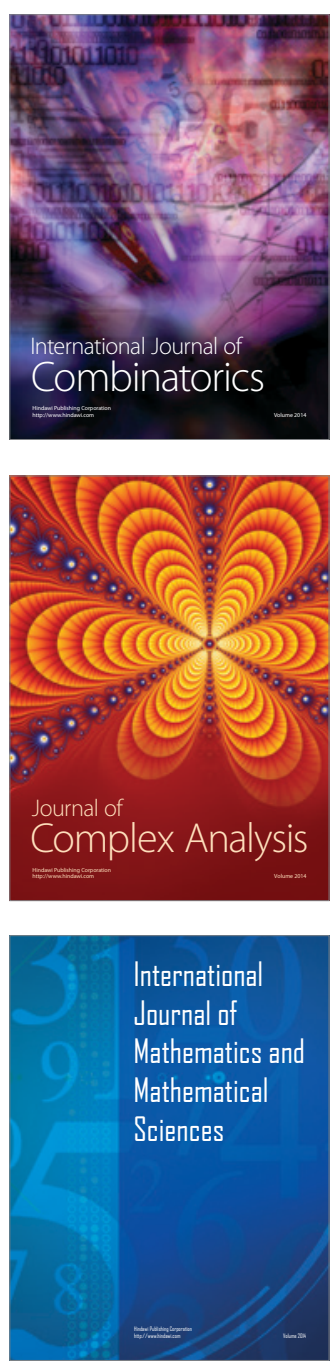
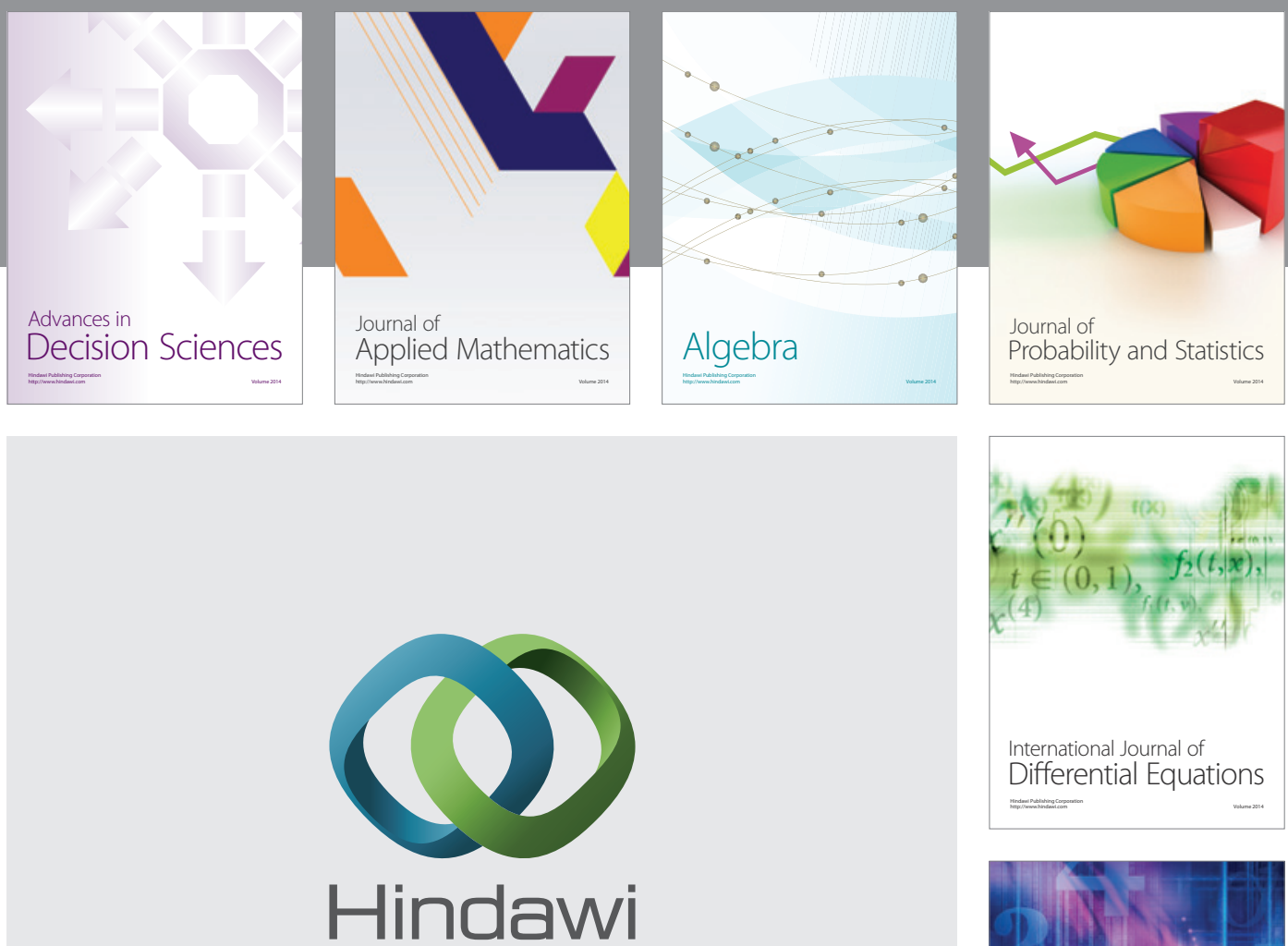

Submit your manuscripts at http://www.hindawi.com
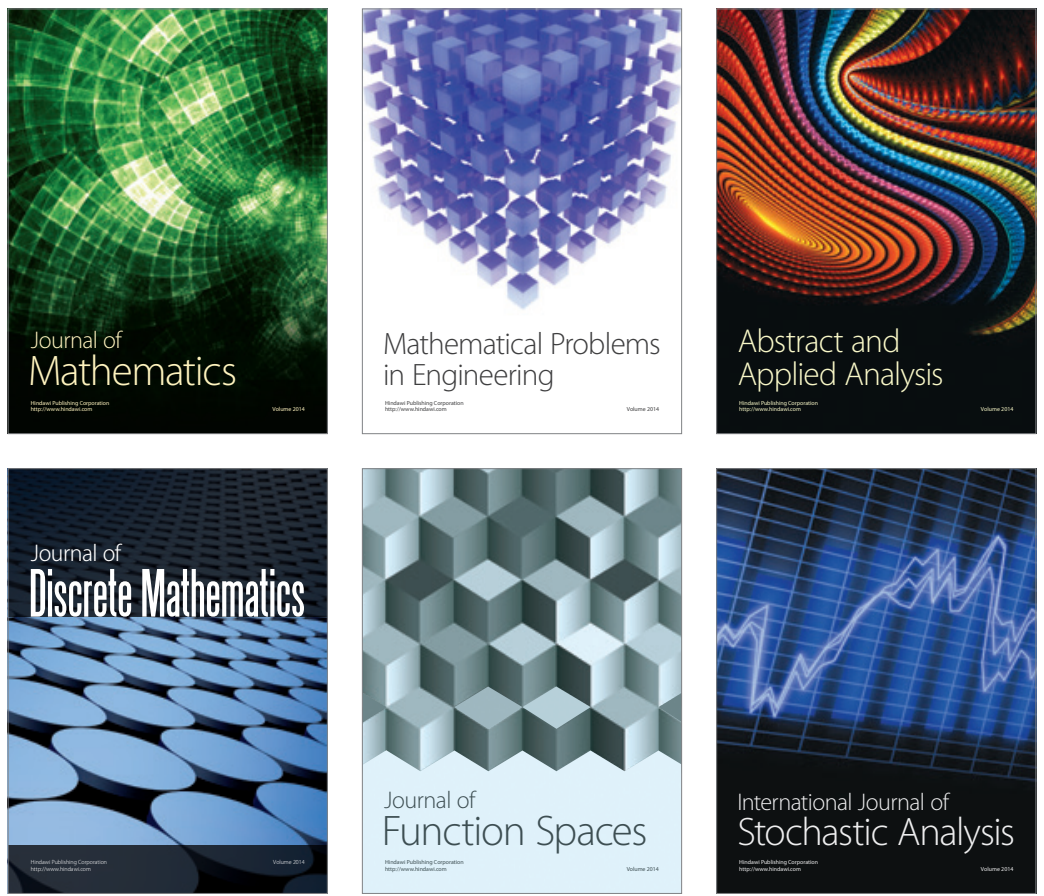

Journal of

Function Spaces

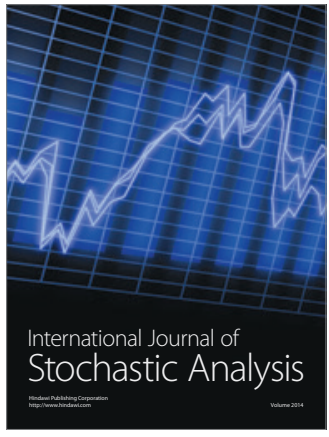

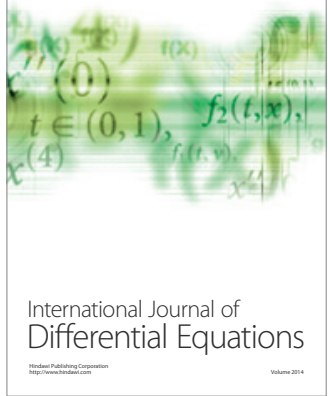
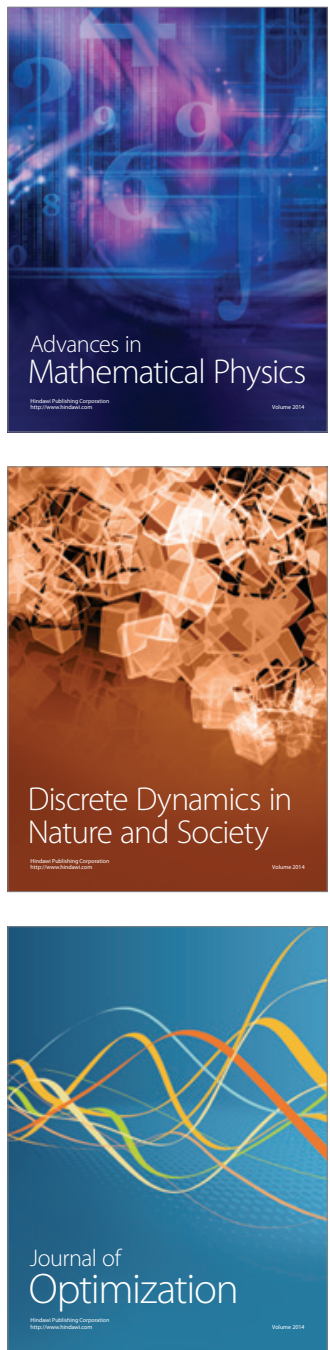